\title{
Processing in a letter-deletion condition
}

\author{
DALE DINNEL and JOHN A. GLOVER \\ The University of Nebraska, Lincoln, Nebraska
}

\begin{abstract}
The availability of context was varied in a letter-deletion manipulation in subjects' reading of a brief passage. The task of supplying missing letters in words presented in the absence of context was more difficult than supplying missing letters for words in context, as determined by study time and number of correct responses. Levels of recall in the noncontext condition, however, were significantly lower than in the context condition, suggesting that the task of supplying missing letters for words in passages requires processing beyond individual lexical items. It seems that the processing required for such tasks is similar to that required by readers when they encounter unfamiliar vocabulary.
\end{abstract}

In recent years, considerable work employing a "difficulty of processing" heuristic has focused on memory for verbal information (e.g., Einstein, 1976; Kolers, 1973; Kolers \& Ostry, 1974; Massan \& Sala, 1978). There have been difficulties, however, in integrating the results of studies on memory in prose research into the framework provided by work on individual words and sentences (see Benton, Glover, \& Bruning, 1983; Benton, Glover, Monkowski, \& Shaughnessy, 1983). For example, Carter (1977) found that subjects who read a scrambled text (a typical difficulty manipulation) recalled text no better than subjects who read a normal version. Schwarz and Flammer (1981) found that a scrambling manipulation resulted in poorer fairy tale recall than did a control condition. Recently, Einstein, McDaniel, Bowers, and Stevens (1984) offered a hypothesis and supporting evidence, based on subjects' incidental recall of prose, that seem to resolve the problem of why difficulty manipulations, such as unscrambling sentences, have not had beneficial effects on readers' memories for prose.

In particular, Einstein et al. (1984) found that a sentence-unscrambling manipulation did enhance memory for prose, but only in conditions in which the relational and sequential aspects of passages were unclear. In addition, they found that a second manipulation, which involved deleting approximately $30 \%$ of the letters in a passage, enhanced recall in a well-structured passage. These results caused Einstein et al. (1984) to postulate that there is an interaction between type of prose (in this case ambiguous vs. well-structured) and type of difficulty manipulation. For instance, the letter-deletion manipulation enhanced incidental recall because it required the processing of individual lexical items, information that is not normally well processed. In contrast, a sentence-unscrambling condition did not enhance subjects' memories for well-structured prose because this aspect of a passage is

Reprints may be obtained from J. A. Glover, Department of Educational Psychology, The University of Nebraska, Lincoln, NE 68588-0440. usually well processed. In essence, then, Einstein et al. (1984) proposed a form of redundancy hypothesis in which only those difficulty manipulations that focus reader attention on nonredundant processing enhance recall.

Einstein et al.'s (1984) work and resulting hypothesis are especially helpful in beginning to resolve some of the inconsistent findings from research on difficulty manipulations during reading. Our own work in this area (see Benton et al., 1983), however, suggests that Einstein et al. may have developed the proper hypothesis but not accurately portrayed the kind of processing required by subjects who must provide letters in mutilated words as they read.

The goal of the current study was to more carefully examine the kinds of processing subjects use when they respond to a letter-deletion manipulation. Our analysis of the letter-deletion manipulation suggested that subjects might very well react to words in which letters are deleted (e.g., f_r e_am _ le) in much the same way that readers react to unfamiliar vocabulary: Subjects' processing would not be specific to the individual lexical items under consideration. Rather, subjects' ability to supply missing letters would involve several context-bound processes, such as predicting the meaning of the word from prior text, employing temporal cues from the text, selecting equivalence cues, and so on. If our analysis is correct, it would indicate that a reader's supplying of missing letters for words in a text enhances the reader's recall because of heightened processing of the context in which the words occur (an elaboration phenomenon, e.g., Reder \& Anderson, 1979) rather than processing of the individual lexical items.

In this experiment, the letter-deletion manipulation was administered to subjects under two conditions-one in which the context of the passage was available and a second in which words were presented serially in the absence of the remainder of the text. Both of these conditions were contrasted with a control condition in which intact words were serially presented to subjects in the absence of the remainder of the text. We predicted that, if our hypothesis about the contextually bound nature of the letter- 
deletion manipulation was correct, then levels of passage recall would be significantly lower in the noncontext condition than in the context condition.

\section{METHOD}

\section{Subjects and Setting}

Subjects were 48 undergraduates enrolled in introductory educational psychology courses who participated in the study for course credit. All testing was conducted with individual subjects in a small work room over a period of six weeks.

\section{Apparatus}

All materials were presented on an Apple IIe microcomputer.

\section{Materials}

The passage was 14 sentences long and described a behavior analyst's experiences in altering the behaviors of a client. ${ }^{1}$ Because all the subjects participating in the experiment had recently completed a unit on behavior analysis, we presumed that this passage would be relatively familiar to them. Two versions of the passage were prepared. In one version, approximately $30 \%$ of the letters were deleted from all words except the one- and two-letter words. The second version was prepared in normal fashion.

\section{Procedures}

Subjects were randomly assigned to one of three conditions: letter deletion, noncontext letter deletion, and noncontext control. In the letter-deletion condition, subjects were shown the entire passage on the computer screen and asked to verbally provide the missing letters for each word as they read. Subjects were informed that they would be tested over the content after they finished reading. Study time and accuracy of letter provision were checked by the experimenter. In the noncontext letter-deletion condition, subjects were presented the same mutilated essay with the same instructions; however, subjects were shown only one word at a time in the passage. They were informed that they could proceed to the next word in the passage by pressing a key and were given practice doing so with a brief sentence dealing with westward expansion. Subjects could not look back at earlier parts of the passage. Again, the experimenter kept track of study time and the correctness of letter provisions. In the control condition, subjects were presented the essay in nonmutilated form one word at a time. Subjects were informed that they could proceed to the next word in the passage by pressing a key and were given practice with the same sentence used in the noncontext letter-deletion condition. Subjects were told that they would be tested over the content when they had completed their reading. Study time was kept by the experimenter. Subjects in all three conditions completed a free recall posttest immediately upon finishing reading.

\section{RESULTS}

The passage was analyzed into 28 propositions, using Kintsch's (1974) analysis of text meaning. The protocols were scored for gist meaning by two independent raters $(R=.92)$. Direct restatements of passage content as well as synonomous terms used to indicate the same meaning were scored as correct recalls. The recall data were then analyzed in a one-way analysis of variance, indicating a significant difference among the conditions $[\mathrm{F}(2,45)=$ $15.77, \mathrm{p}<.01]$. The Tukey procedure $(\alpha=.05)$ was then used to follow up the analysis of variance and indicated that the letter deletion with context condition $(M=$ $8.06, \mathrm{SD}=2.05$ ) resulted in significantly superior recall than either the control condition $(M=6.94, S D=1.73)$ or the noncontext letter-deletion condition $(M=4.56$, $\mathrm{SD}=1.59)$. In addition, subjects in the control condition recalled significantly more than subjects in the noncontext letter-deletion condition.

Because we were separately interested in study time as an index of the "difficulty" of processing, these data were analyzed in a separate analysis of variance, indicating a significant difference among conditions $[\mathrm{F}(2,45)=$ $108.66, \mathrm{p}<.01]$. The Tukey procedure $(\alpha=.05)$ indicated that the noncontext letter-deletion condition $(M=$ $678.57 \mathrm{sec}, \mathrm{SD}=109.24 \mathrm{sec}$ ) required significantly more study time than either the letter deletion with context condition $(M=422.71 \mathrm{sec}, \mathrm{SD}=152.93 \mathrm{sec})$ or the control condition $(M=107.40 \mathrm{sec}, \mathrm{SD}=28.90 \mathrm{sec})$. In addition, the letter-deletion condition with context required significantly more study time than the control condition.

A check on the accuracy of subjects' provision of letters in the two experimental conditions was also performed. The results indicated that about $96 \%$ of the deleted letters were correctly replaced in the deleted letters with context condition. In contrast, only $86 \%$ of the letters in the noncontext deleted letters condition were correctly replaced.

\section{DISCUSSION}

The results suggest that context plays an important role in the letter-deletion manipulation. Recall of passage content was nearly twice as great in the letter-deletion condition with context than it was in the noncontext letter-deletion condition. The difference in the accuracy with which subjects supplied missing letters in these two conditions also suggests that the absence of context strongly affects subjects' processing. Readers' need for context in order to perform the processing necessary for the letter-deletion task is also seen in the fact that the noncontext control condition resulted in significantly greater levels of recall than the noncontext letterdeletion condition. The study time data, here employed as a check on the difficulty of the separate tasks, seem to indicate that the noncontext letter-deletion task was indeed far more difficult than the letter deletion with context task. These results imply that a difficulty hypothesis is inadequate to deal with the results of processing in which subjects must supply missing letters for words in passages.

The overall results seem to indicate that the processing required of subjects in a letter-deletion condition is broader than Einstein et al. (1984) concluded and that the processing includes the use of contextual information to supply missing letters. Although the redundancy hypothesis suggested by Einstein et al. (1984) has intuitive appeal, it seems necessary to reexamine their results in light of the type of processing that appears to be involved in the letter-deletion manipulation, because more than individual lexical item-specific processing occurs. It would seem that a version of an elaboration hypothesis (e.g., Reder \& Anderson, 1979) might best describe the processing seen in letter-deletion tasks.

\section{REFERENCES}

Benton, S. L., Glover, J. A., \& Bruning, R. H. (1983). The effect of number of decisions on prose recall. Journal of Educational Psychology, 75, 382-390.

Benton, S. L., Glover, J. A., Monkowski, P. G., \& ShaughNESSY, M. (1983). Decision difficulty and recall of prose. Journal of Educational Psychology, 75, 727-742.

Carter, J. F. (1977). Comments on chapter 6 by Meyer. In R. C. 
Anderson, R. J. Spiro, \& W. E. Montague (Eds.), Schooling and the acquisition of knowledge (pp. 201-208). Hillsdale, NJ: Erlbaum. EINSTEIN, G. O. (1976). Effects of simultaneous interference upon free recall learning and retention. Memory \& Cognition, 4, 701-708.

Einstein, G. O., McDaniel, M. A., Bowers, C. A., \& Stevens, D. T. (1984). Memory for prose: The influence of relational and propositionspecific processing. Journal of Experimental Psychology: Learning, Memory, \& Cognition, 10, 133-143.

KINTSCH, W. (1974). The representation of meaning. Hillsdale, NJ: Erlbaum.

Kolers, P. A. (1973). Remembering operations. Memory \& Cognition, 1, 347-355.

Kolers, P. A., \& OSTRY, D. J. (1974). Time course of loss of information regarding pattern analyzing operations. Journal of Verbal Learning \& Verbal Behavior, 13, 599-612.
MASSAN, M. E. J., \& SALA, L. S. (1978). Interactive processes in sentence comprehension and recognition. Cognitive Psychology, 10, 244-270.

Reder, L. M., \& ANDERson, J. R. (1979). A comparison of texts and their summaries. Memorial consequences. Journal of Verbal Learning \& Verbal Behavior, 19, 121-134.

SChWARZ, M. N. K., \& Flammer, A. (1981). Text structure and titleeffects on comprehension and recall. Journal of Verbal Learning \& Verbal Behavior, 20, 61-66.

\section{NOTE}

1. Copies of the passage are available upon request.

(Manuscript received for publication March 19, 1985.) 